\section{A posteriori odds}

R.-D. Hilgers ${ }^{1}$, N. Heussen ${ }^{1}$ und S. Stanzel ${ }^{2}$

${ }^{1}$ Institut für Medizinische Statistik, Universitätsklinikum der RWTH Aachen, Aachen, Deutschland

${ }^{2}$ DKFZ Heidelberg, Heidelberg, Germany

Synonym(e) Nachtest odds

Englischer Begriff a posteriori odds

Definition Das a posteriori odds gibt das Verhältnis der Wahrscheinlichkeit an, dass eine Person mit positivem Testergebnis auch tatsächlich erkrankt ist, zur Wahrscheinlichkeit, dass eine Person mit positivem Testergebnis tatsächlich nicht erkrankt ist.
Beschreibung Das a posteriori odds wird üblicherweise geschätzt durch den Quotienten

$$
\frac{\frac{a}{a+b}}{1-\frac{a}{a+b}}
$$

(Bezeichnungen s. Tabelle im Stichwort $\gg$ Vierfeldertafel).

\section{Literatur}

Hilgers R-D, Bauer P, Scheiber V (2002) Einführung in die Medizinische Statistik. Springer, Berlin/Heidelberg/New York 